\title{
Age-based vs. stock level control policies for a perishable inventory system
}

\author{
Eylem Tekin ${ }^{\mathrm{a}}$, Ülkü Gürler ${ }^{\mathrm{b}, *}$, Emre Berk $^{\mathrm{c}}$ \\ ${ }^{a}$ Department of Industrial Engineering and Management Science, Northwestern University, Evanston, IL 60208-3119, USA \\ ${ }^{\mathrm{b}}$ Department of Industrial Engineering, Bilkent University, 06533 Ankara, Turkey \\ ${ }^{\mathrm{c}}$ Faculty of Business Administration, Bilkent University, 06533 Ankara, Turkey
}

Received 31 August 1999; accepted 24 July 2000

\begin{abstract}
In this study, we investigate the impact of modified lotsize-reorder control policy for perishables which bases replenishment decisions on both the inventory level and the remaining lifetimes of items in stock. We derive the expressions for the key operating characteristics of a lost sales perishable inventory model, operating under the proposed age-based policy, and examine the sensitivity of the optimal policy parameters with respect to various system parameters. We compare the performance of the suggested policy to that of the classical $(Q, r)$ type policy through a numerical study over a wide range of system parameters. Our findings indicate that the age-based policy is superior to the stock level policy for slow moving perishable inventory systems with high service levels. (C) 2001 Elsevier Science B.V. All rights reserved.
\end{abstract}

Keywords: Inventory; Perishable; Lost sales

\section{Introduction and literature review}

In this paper, we consider a continuous review perishable inventory system operating under a modified lotsize-reorder control policy which also takes into account the remaining lifetime of the items in stock. Due to the importance of perishable inventories in food, chemical and pharmaceutical industries, mathematical modeling of such systems have attracted the attention of many researchers.

Under periodic review, if the items cannot be retained in stock for more than one period, the perishable inventory problem reduces to the well-known newsvendor problem. When the lifetime of the items is greater than one period, the problem becomes more difficult. Van Zyl [30] provides the earliest formulation

\footnotetext{
${ }^{*}$ Corresponding author. Tel.: +90-312-2901520; fax: +90-312-2664054.

E-mail addresses: eylem@nwu.edu (E. Tekin), ulku@bilkent.edu.tr (U. Gürler), eberk@bilkent.edu.tr (E. Berk).
} 
for finite horizon, where the shelflife is assumed to be exactly two review periods. Following Van Zyl, Nahmias and Pierskalla [14], Fries [3] and Nahmias [15] investigate the properties of the optimal policy for the two-period and the general $m$-period shelflives, respectively. Given the complexity of the optimal policy structure for lifetimes longer than two periods, research has henceforth shifted towards development of approximate policies. Among them, we may cite Nahmias [16], Cohen [2], Nahmias [17], Nahmias [18] and recently Nandsakumar and Morton [21]. A comprehensive survey of works under periodic review can be found in Nahmias [19].

The existing work under continuous review can be categorized as models with zero, exponential and constant leadtimes. With zero leadtime, Weiss [31] considers a model with Poisson external demand process and constant shelflives for the items in a batch, and shows that the optimal policy is a continuous review $(s, S)$ policy. Kalpakam and Arivarignan [5] consider the $(s, S)$ model with exponential shelflives and a zero reorder point. Liu [8] studies a similar model with backorders. Recently, Lian and Liu [10] have studied a discrete time $(s, S)$ backordering model with geometric interdemand times and batch demands. Liu and Lian [11] consider a similar model with constant shelflives and a renewal demand process. Liu and Shi [9] later investigate the exponential lifetime case in the same setting. Moorthy et al. [13] consider a different model, where the lifetimes are Erlangian but the aging process of an item begins only after it is put on display. With exponential leadtimes, Kalpakam and Sapna [6] analyze an $(s, S)$ system with Poisson demands and exponential shelflives. Excess demand is lost and only one outstanding order is allowed at any point in time. Liu and Yang [12] consider backordering and relax the restriction on the number of outstanding orders in a model similar to Kalpakam and Sapna [6]. Kalpakam and Sapna [7] consider a lost sales $(S-1, S)$ model with exponential lifetimes and demands generated according to a general renewal process. With instantaneous replenishment and exponential leadtimes, mostly, it is possible to analyze the perishable inventory systems by means of Markov renewal techniques. With constant leadtimes, a Markovian structure can not be attained and the analysis becomes significantly difficult. For constant leadtimes, Schmidt and Nahmias [27] study $(S-1, S)$ policies when external demands are generated from a Poisson process, items have fixed shelflives and excess demand is lost. The steady-state distribution of stocking levels is obtained by means of the method of supplementary variables. This is the first study that provides the exact continuous review analysis of perishables with fixed life in the presence of nonzero leadtimes. Recently Perry and Posner [22], have considered an $(S-1, S)$ model with constant leadtimes, leadtime-dependent backordering and analyze it by means of level-crossing methodology. Nahmias and Wang [20] provide the first treatment of perishable inventory with fixed ordering costs and a constant leadtime. For Poisson demand, a heuristic $(Q, r)$ model is developed under the assumption of at most one order outstanding. The difficulty of fixed lifetimes is circumvented by means of a constant decay approximation for the aging process. Chiu [1] re-examines the problem and provides another heuristic solution. Ravichandran [25] considers a constant lifetime $(s, S)$ system with Poisson demand and random leadtimes. He introduces a specific aging process for each order batch similar to that in [13].

There is also a large body of work on continuously deteriorating inventory systems, which falls outside the scope of this study. For an exhaustive review, we refer the reader to [24].

The form of the optimal policy for a continuous review perishable inventory system with positive leadtime appears to be extremely complex for analysis and implementation. Hence, as summarized above, the existing studies consider only the $(s, S)$ type control policies, which are known to be optimal for nonperishables facing Markovian demand. In perishable inventory systems, the effective demand is nonMarkovian due to decaying of items even with Markovian external demand. Schultz [28] has shown for slow moving nonperishable items that time-based ordering policies dominate the conventional inventory level-based policies when the interdemand distribution has an increasing hazard rate. Therefore, it is worthwhile to investigate the performance of similar age-based ordering policies for perishables. The contribution of this study is to introduce such a control policy which bases reordering decisions not only on the stock levels but also on the remaining shelflives of the items in stock. Our results show that the proposed 
age-based policy dominates the classical control policies and suggest that policies of the proposed structure would be promising in other perishable inventory settings as well. We hope that our findings would initiate research in this direction.

The rest of the paper is organized as follows. Section 2 states the assumptions of the model and introduces the control policy. In Section 3, we develop the expressions for the key operating characteristics of the inventory system, and discuss the special cases of the proposed model. In Section 4, numerical results are presented on the sensitivity of the optimal policy parameters with respect to various system parameters and on the performance of the proposed policy in comparison with the classical policies. The paper ends with some concluding remarks and comments on future work.

\section{The model}

We consider an inventory system facing unit external demands generated by a Poisson process with mean $\lambda$. All unmet demands are lost and there is a positive leadtime, $L$. We assume that the items in a batch are perishable, with a constant lifetime $\tau$, under a special aging process as explained below. The associated costs are the fixed ordering cost $K$ incurred with each order, the holding cost charged at $h$ per unit held in stock per unit time and the perishing cost $p$ charged per unit that perishes in stock. There is no direct cost of lost sales. Instead, we impose a service level constraint that requires the fraction of unmet demand not to exceed a prespecified value, $\alpha$.

We assume that the aging of items in a batch begins after all units of the previous batch are exhausted either by demand or through decay. That is, the aging of a batch starts when the batch is 'unpacked' for consumption. This aging phenomenon was observed by the authors in the production processes of an aircraft manufacturer. In this application, composite materials are used for the manufacture of aircraft body panels. The composite fabrics in use are perishable and have a lifetime which depends on the storage environment. Before being released to the shop floor for cutting and plastering, the fabric is kept in a special freezer without any deterioration up to six months. Given the low volume of consumption and the long lifetime in the freezers, composite fabrics are virtually nonperishable materials as long as they are stored at very low temperatures. As the composite materials are released to the shop floor, deterioration starts due to environmental exposure; hence, the composite fabrics have a very short floorlife (life time on the shop floor) measured in weeks. Once the materials are sent out to the production area, they stay in this severe environment until they perish or are consumed. Other examples of perishable goods that experience a similar aging process are some of the adhesives and coatings used in aircraft and shipbuilding industries, and foodstuffs in supermarkets that are kept in separate storage areas prior to being put on shelves. (See also $[13,25]$ for the same aging process.) Note that this special aging assumption is essentially similar to the commonly made assumption that items in an order do not deteriorate while in transit. Limited simulation studies we conducted, also indicate that the model developed herein is a good approximation to slow moving inventory systems in which items begin aging as soon as they join the stock. With this aging structure, we propose the following modified lotsize reorder control policy which bases the replenishment decision on both the inventory levels and the remaining shelflives of the items in stock.

Policy. A replenishment order of size $Q$ is placed either when the inventory drops to $r$, or when $T$ units of time have elapsed since the last instance at which the inventory level hit $Q$, whichever occurs first.

The policy described above is in spirit a modified reorder point-ordering quantity $(Q, r)$ policy [23] and, therefore, we refer to it as the $(Q, r, T)$ policy. Further, due to the specified aging process, $T$ corresponds directly to an age threshold for reorder, whereas, $r$ is an inventory level threshold for reorder in the classical sense. We assume in the sequel that $r<Q$, which results in at most one outstanding order at any time given the assumption of lost sales. 
Under the above policy, it is easy to see that the instances at which the inventory level hits $Q$ constitute regenerative epochs. Hence, we define a cycle as the time between two such consecutive instances, and employ the renewal reward theorem [26] to derive the operating characteristics of the system. Our objective is to find the optimal parameters of the control policy that minimizes the expected total cost rate under the service level constraint.

\section{Operating characteristics}

In this section, we derive the expressions for the expectations of cycle length, on hand inventory, number of lost sales and number of items that perish in a cycle as a function of the policy parameters $Q, r$ and $T$. These expressions are then used to construct the average cost function with the service level criterion. Before we proceed with the derivations, we introduce some notation. Let $X_{n}, n=1,2, \ldots$, denote the random variable representing the arrival time of $n$th demand with distribution function $F_{n}(t)$ and let $f(t)$ be the p.d.f. of the time interval between successive demands. Also, let $N(t)$ denote the counting process associated with the demand process in $(0, t)$, such that $F_{n}(t)$ is the probability that $N(t) \leqslant n$, and let $\bar{F}_{n}(t)=1-F_{n}(t)$.

As illustrated in Figs. 1 and 2, there exist eight possible realizations for a cycle, based on the relations among the age threshold for reorder, the time of the $(Q-r)$ th demand, the time of the $Q$ th demand, and the shelflife. Note here that $X_{Q-r}$ and $X_{Q}$ are random variables, whereas $T$ and $\tau$ are nonnegative constants. Fig. 1 illustrates the realizations where the order placement is triggered by the age threshold, that is,
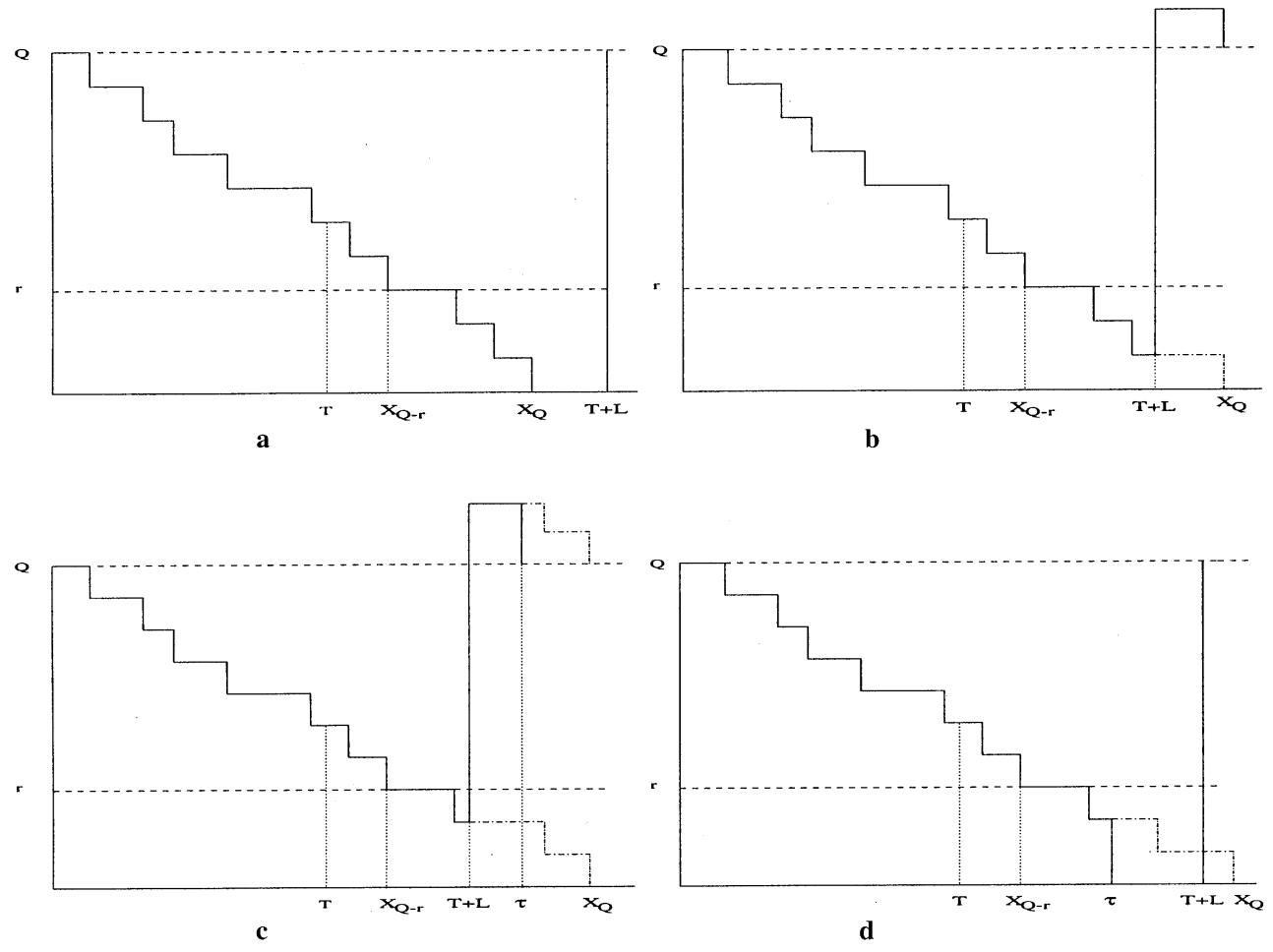

Fig. 1. Possible inventory level realizations in a cycle $\left(T<X_{Q-r}\right)$. 


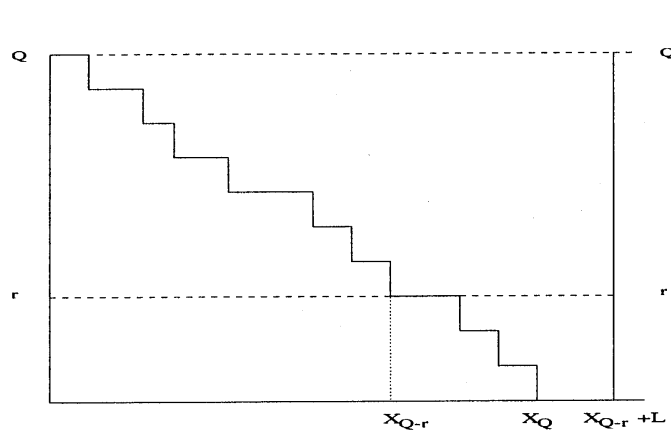

a

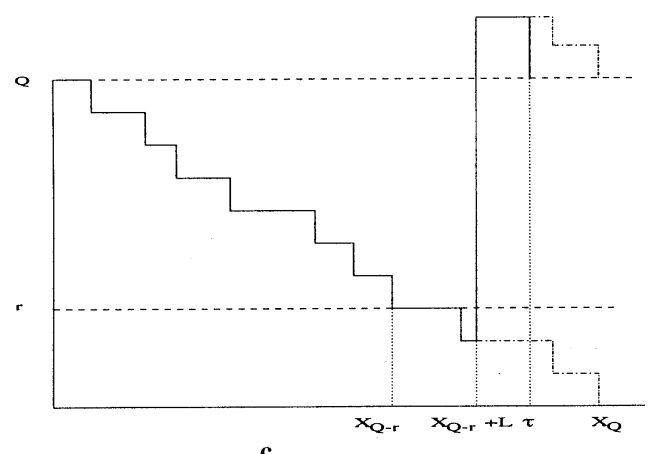

c

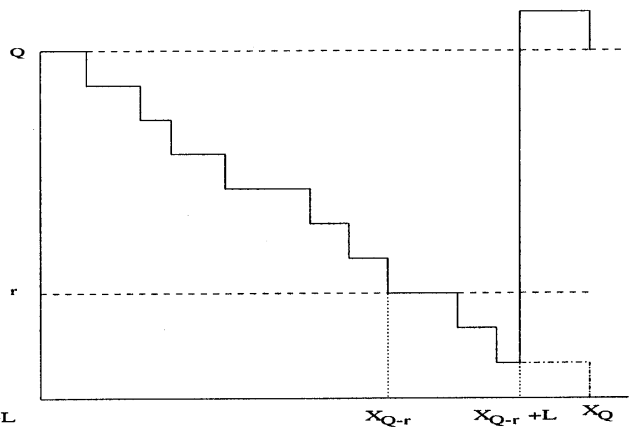

b

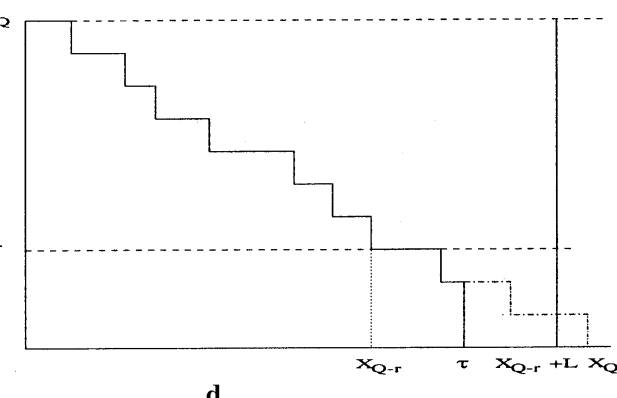

d

Fig. 2. Possible inventory level realizations in a cycle $\left(T>X_{Q-r}\right)$.

when $T<X_{Q-r}$, whereas Fig. 2 illustrates the cases where an order is placed when the inventory level drops to $r$ before perishing, that is, $T>X_{Q-r}$. In the following analysis, we do not allow $T$ to be greater than $\tau$, since it would not be desirable to postpone the ordering decision until a batch has completely deteriorated. For the sake of clarity, we consider the cases $\tau>T+L$ and $T<\tau<T+L$ separately. Derivations of the expressions in this section are given in the Appendix A.1.

Case 1. $T<\tau<T+L$.

Considering all possible realizations for a cycle and combining some events, we express the cycle length, $C L$, as follows:

$$
C L= \begin{cases}X_{Q-r}+L & \text { if } X_{Q-r}<T, X_{Q}<\min \left(X_{Q-r}+L, \tau\right) \\ & \text { or } X_{Q-r}<T, \tau<\min \left(\tau, X_{Q-r}+L\right), \\ X_{Q} & \text { if } X_{Q-r}<T, X_{Q-r}+L<X_{Q}<\tau, \\ \tau & \text { if } X_{Q-r}<\tau-L, X_{Q}>\tau \\ T+L & \text { if } T<X_{Q-r}<\tau\end{cases}
$$

Then the expected cycle length is found as follows:

$$
\begin{aligned}
E[C L]= & L+T \bar{F}_{Q-r}(T)+\frac{Q-r}{\lambda}\left[F_{Q-r+1}(T)-F_{Q-r+1}(\tau-L)\right] \\
& +\left[\tau-L \bar{F}_{r}(L)-\frac{r}{\lambda} F_{r+1}(L)\right] F_{Q-r}(\tau-L)+\eta(Q, r, T),
\end{aligned}
$$


where

$$
\eta(Q, r, T)=\int_{0}^{\tau-L}\left[\frac{r}{\lambda} F_{r+1}(\tau-t)-(\tau-t) F_{r}(\tau-t)\right] f_{Q-r}(t) \mathrm{d} t
$$

is the expected time that a received batch stays in stock until the previous one perishes or is depleted. We denote the area under the inventory level curve within a cycle as $O H$. Considering the possible realizations mentioned above, it can be expressed as

$$
O H= \begin{cases}\sum_{i=1}^{Q} X_{i} & \text { if } X_{Q-r}<T, X_{Q}<\min \left(X_{Q-r}+L, \tau\right) \\ & \text { or } X_{Q-r}>T, X_{Q}<\tau, \\ \sum_{i=1}^{N(\tau)} X_{i}+\tau[Q-N(\tau)] & \text { if } \tau<X_{Q}, \tau-L<X_{Q-r}<T, \\ & \text { or } \tau<X_{Q}, T<X_{Q-r}, \\ \sum_{i=1}^{Q} X_{i}+Q\left[X_{Q}-X_{Q-r}-L\right] & \text { if } X_{Q-r}<T, X_{Q-r}+L<X_{Q}<\tau, \\ \sum_{i=1}^{N(\tau)} X_{i}+\tau[Q-N(\tau)] & \\ +Q\left(\tau-X_{Q-r}-L\right) & \text { if } X_{Q-r}<\tau-L, X_{Q}>\tau .\end{cases}
$$

Taking expectations, we obtain the following expression:

$$
\begin{gathered}
E[O H]=Q\left\{\eta(Q, r, T)+\frac{Q+1}{2 \lambda} F_{Q+1}(\tau)+\tau \bar{F}_{Q}(\tau)+\left[\tau-L \bar{F}_{r}(L)-\frac{r}{\lambda} F_{r+1}(L)\right] F_{Q-r}(\tau-L)\right. \\
\left.-\frac{Q-r}{\lambda} F_{Q-r+1}(\tau-L)\right\}-\frac{\lambda \tau^{2}}{2} \bar{F}_{Q-1}(\tau) .
\end{gathered}
$$

Similarly, the number of lost sales in a cycle, $L S$, is

$$
L S= \begin{cases}N\left(X_{Q-r}+L-X_{Q}\right) & \text { if } X_{Q-r}<T, X_{Q-r}<X_{Q}<X_{Q-r}+L, X_{Q}<\tau, \\ N\left(X_{Q-r}+L-\tau\right) & \text { if } \tau-L<X_{Q-r}<T, X_{Q}>\tau \\ N(T+L-\tau) & \text { if } X_{Q-r}>T, X_{Q}>\tau \\ N\left(T+L-X_{Q}\right) & \text { if } T<X_{Q-r}<X_{Q}<\tau\end{cases}
$$

Then the expected number of lost sales in a cycle is

$$
\begin{aligned}
E[L S]= & \lambda\left[\eta(Q, r, T)-\tau \bar{F}_{Q}(\tau)-T \bar{F}_{Q-r}(T)+L\right]+\lambda\left[\tau-L \bar{F}_{r}(L)-\frac{r}{\lambda} F_{r+1}(L)\right] F_{Q-r}(\tau-L) \\
& +(Q-r)\left[F_{Q-r+1}(T)-F_{Q-r+1}(\tau-L)\right]-Q F_{Q+1}(\tau) .
\end{aligned}
$$

Finally, the number of items that perish in a cycle, $P$, is given by

$$
P= \begin{cases}Q-N(\tau) & \text { if } X_{Q}>\tau \\ 0 & \text { otherwise }\end{cases}
$$

and

$$
\begin{aligned}
E[P] & =E\left[(Q-N(\tau)) I\left(X_{Q}>\tau\right)\right] \\
& =Q \bar{F}_{Q}(\tau)-\sum_{n=0}^{Q-1} n P(N(\tau)=n)=Q \bar{F}_{Q}(\tau)-\lambda \tau \bar{F}_{Q-1}(\tau) .
\end{aligned}
$$


Case 2. $\tau>T+L$.

The operating characteristics for this case are derived similarly and the following expressions are obtained:

$$
E[C L]=\zeta(Q, r, T)+\tau \bar{F}_{Q}(\tau)+\frac{Q}{\lambda} F_{Q+1}(\tau)
$$

where

$$
\begin{aligned}
\zeta(Q, r, T)= & \int_{T}^{T+L}\left[(T+L-t) F_{r}(T+L-t)-\frac{r}{\lambda} F_{r+1}(T+L-t)\right] f_{Q-r}(t) \mathrm{d} t \\
& +\left[L F_{r}(L)-\frac{r}{\lambda} F_{r+1}(L)\right] F_{Q-r}(T)
\end{aligned}
$$

is the expected duration of a stockout period. Also, we have

$$
\begin{aligned}
E[O H]= & Q\left\{\zeta(Q, r, T)+T F_{Q-r}(T)-\frac{Q-r}{\lambda} F_{Q-r+1}(T)+\frac{3 Q+1}{2 \lambda} F_{Q+1}(\tau)+2 \tau \bar{F}_{Q}(\tau)-T-L\right\} \\
& -\frac{\lambda \tau^{2}}{2} \bar{F}_{Q-1}(\tau)
\end{aligned}
$$

and

$$
\begin{aligned}
& E[L S]=\lambda \zeta(Q, r, T) \\
& E[P]=Q \bar{F}_{Q}(\tau)-\lambda \tau \bar{F}_{Q-1}(\tau) .
\end{aligned}
$$

We can now formulate the optimization problem of minimizing the expected total cost rate, $T C(Q, r, T)$, subject to the service level constraint

$$
\min T C(Q, r, T)=\frac{K+h E[O H]+p E[P]}{E[C L]}
$$

s.t.

$$
\frac{E[L S]}{\lambda E[C L]} \leqslant \alpha
$$

where $\alpha$ is the maximum permissible fraction of lost sales. Note that from the theory of Lagrange multipliers, minimizing (13) subject to (14) is equivalent to minimizing a modified long-run average cost with explicit shortage costs. The foregoing expressions regarding the cost functions render it almost impossible to derive analytical results about the average cost function. The implementations in the rest of the manuscript are, therefore, done via numerical tools.

Special cases.

(i) $(Q, r)$ Model. If we let $T=\tau$ in the original model, we obtain a $(Q, r)$ type model for perishables, in which an order is placed when the inventory position hits $r$ or when all items in stock perish at age $\tau$ (raising the inventory level to $Q$ ). Perishing would not be observed too often due to its high costs in realistic settings; therefore, the reordering decisions under this policy would typically be based on only stocking level. We use this model in our numerical study to compare the performances of the age-based policy and the conventional $(Q, r)$ type policy. As another case, note that our perishable age-based policy reduces to the classical $(Q, r)$ policy for nonperishables [4] as $\tau \rightarrow \infty$, and $T \rightarrow \infty$, respectively. 
(ii) $(Q, T)$ Model. Another two-parameter policy is obtained as a special case when we let $r=0$ in our model. Noting again that perishing would not be common in realistic cost settings, the reordering decisions under this policy would typically be based only on the age of the batch currently in use. We have conducted a numerical study between the $(Q, r)$ and $(Q, T)$ policies and observed that the $(Q, r)$ policy dominated the $(Q, T)$ over the experimental set we investigated [29]. Hence, we shall not elaborate further on this special case.

\section{Numerical results}

We conducted numerical studies to examine the sensitivity of the optimal policy parameters with respect to various system parameters, and to compare the performance of the $(Q, r, T)$ policy vis-à-vis the classical $(Q, r)$ policy over a wide range of system parameter settings. To be consistent in our comparison of the two classes of control policies, we maintain the restriction on $r(r<Q)$ for both policies.

\subsection{Sensitivity analysis}

We first discuss our findings about how the optimal policy parameters and average cost rate change with respect to ordering cost, perishing cost, permissible fraction of lost sales and shelflife. The reported experimental points represent a broad range of cases from almost no lost sales with high ordering costs and short lifetimes to lost sales of $10 \%$ with low ordering costs and long shelflives (see Table 1). Disposal costs of toxic raw materials like adhesives and composite materials are very high due to strict environmental regulations; very high unit costs of perishing $(p=50)$ in our numerical set are intended to include such scenarios.

We present our results in Table 2 for fixed values of $\lambda=5, h=1$ and $L=1$. We note that most of the results agree with what one would intuitively expect.

The optimal ordering quantity $Q^{*}$ increases as $K$ increases. However, this increase is not as significant as it is for nonperishables. For instance, when the shelflife is short $(\tau=2)$, there is almost no change in $Q^{*}$ as $K$ increases. The overall effect of increasing $K$ on reordering is to delay the order placement by either increasing $T^{*}$ or decreasing $r^{*}$ (or both).

As the perishing cost $p$ increases, we observe that $Q^{*}$ decreases in order to decrease the average number of items that perish. At the same time, the reordering decisions get to be made sooner, that is at smaller $T^{*}$ or larger $r^{*}$. When products are subject to decay in a short time and a small fraction of lost sales is desired $(\alpha<0.02), r^{*}$ is not sensitive to the changes in $p$, but $T^{*}$ decreases considerably as $p$ increases. The expected cost rate is observed to be more sensitive to $T$ when $\alpha$ and $\tau$ values are small.

The length of the shelflife has a noticeable effect on the optimal ordering quantity. As the shelflife increases, $Q^{*}$ increases considerably. At the same time, $r^{*}$ tends to decrease slightly whereas $T^{*}$ tends to

Table 1

Test parameters

\begin{tabular}{lll}
\hline Parameter & Symbol & Values tested \\
\hline Frac. of lost sales & $\alpha$ & $0.005,0.01,0.02,0.05,0.1$ \\
Ordering cost & $K$ & 50,100 \\
Perishing cost & $p$ & $1,10,50$ \\
Shelf life & $\tau$ & $2,4,6,12,15,20$ \\
Demand rate & $\lambda$ & $0.25,0.5,1,2,5,10$ \\
\hline
\end{tabular}




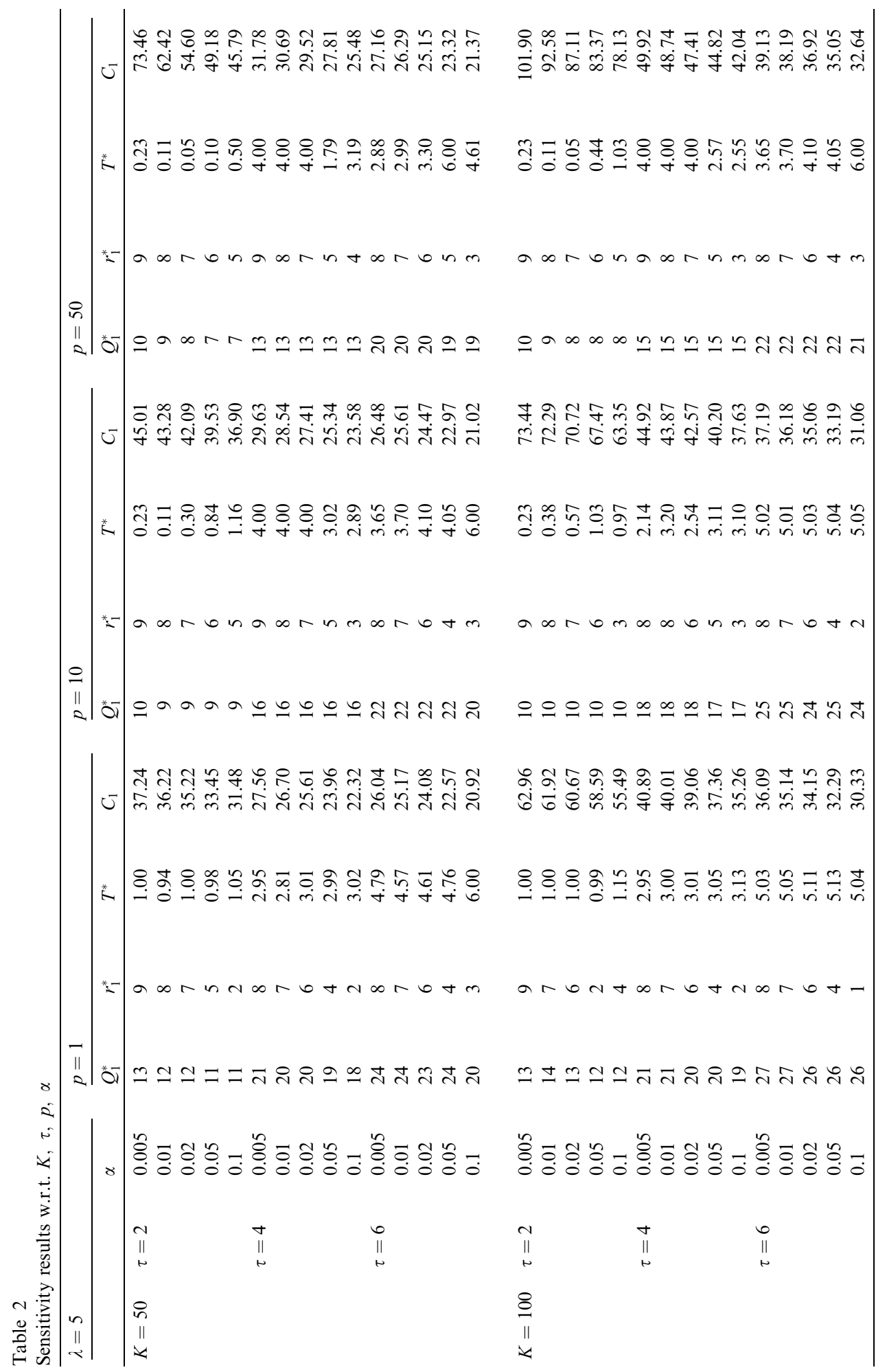


Table 3

Sensitivity results w.r.t. $L, p, \alpha$

\begin{tabular}{|c|c|c|c|c|c|c|c|c|c|c|c|c|c|}
\hline \multicolumn{2}{|l|}{$\lambda=5$} & \multicolumn{4}{|c|}{$p=1$} & \multicolumn{4}{|c|}{$p=10$} & \multicolumn{4}{|c|}{$p=50$} \\
\hline$\tau=2$ & $\alpha$ & $\overline{Q_{1}^{*}}$ & $r_{1}^{*}$ & $T^{*}$ & $C_{1}$ & $\overline{Q_{1}^{*}}$ & $r_{1}^{*}$ & $T^{*}$ & $C_{1}$ & $\overline{Q_{1}^{*}}$ & $r_{1}^{*}$ & $T^{*}$ & $C_{1}$ \\
\hline \multirow{5}{*}{$L=0.5$} & 0.005 & 12 & 5 & 1.50 & 36.12 & 9 & 5 & 0.82 & 43.09 & 7 & 5 & 0.28 & 52.93 \\
\hline & 0.01 & 12 & 4 & 1.50 & 35.39 & 9 & 5 & 1.59 & 42.18 & 7 & 5 & 2.00 & 51.84 \\
\hline & 0.02 & 12 & 3 & 1.50 & 34.77 & 9 & 4 & 1.52 & 40.93 & 7 & 4 & 0.77 & 50.76 \\
\hline & 0.05 & 11 & 2 & 1.52 & 33.40 & 9 & 3 & 1.58 & 39.10 & 7 & 3 & 1.11 & 48.36 \\
\hline & 0.1 & 11 & 0 & 1.58 & 31.49 & 9 & 1 & 1.45 & 36.64 & 7 & 2 & 1.34 & 45.30 \\
\hline \multirow[t]{5}{*}{$L=1$} & 0.005 & 13 & 9 & 1.00 & 37.24 & 10 & 9 & 0.23 & 45.01 & 10 & 9 & 0.23 & 73.46 \\
\hline & 0.01 & 12 & 8 & 0.94 & 36.22 & 9 & 8 & 0.11 & 43.28 & 9 & 8 & 0.11 & 62.42 \\
\hline & 0.02 & 12 & 7 & 1.00 & 35.22 & 9 & 7 & 0.30 & 42.09 & 8 & 7 & 0.05 & 54.60 \\
\hline & 0.05 & 11 & 5 & 0.98 & 33.45 & 9 & 6 & 0.84 & 39.53 & 7 & 6 & 0.10 & 49.18 \\
\hline & 0.1 & 11 & 2 & 1.05 & 31.48 & 9 & 5 & 1.16 & 36.90 & 7 & 5 & 0.50 & 45.79 \\
\hline \multirow[t]{5}{*}{$L=1.5$} & 0.005 & 13 & 12 & 0.04 & 38.41 & 13 & 12 & 0.04 & 53.79 & 13 & 12 & 0.04 & 122.12 \\
\hline & 0.01 & 14 & 11 & 0.46 & 37.06 & 12 & 3 & 0.03 & 49.17 & 12 & 3 & 0.03 & 102.09 \\
\hline & 0.02 & 13 & 10 & 0.49 & 35.56 & 11 & 10 & 0.04 & 45.05 & 11 & 10 & 0.04 & 84.27 \\
\hline & 0.05 & 12 & 7 & 0.50 & 33.50 & 10 & 9 & 0.25 & 40.68 & 10 & 9 & 0.25 & 67.86 \\
\hline & 0.1 & 11 & 5 & 0.54 & 31.50 & 9 & 8 & 1.13 & 37.13 & 9 & 8 & 1.13 & 54.53 \\
\hline
\end{tabular}

increase. It seems that the effect of the age threshold decreases with increasing shelflife. As expected, the average costs are increasing in $p$ and $K$ but decreasing in $\tau$ and $\alpha$.

For the inventory system under consideration, the ordering decision depends not only on the demand rate but also on the lifetime of items. In order to avoid lost sales during the leadtime, the perishing time is as important as the number of demands during the leadtime. Thus, we are particularly interested in the interaction of the leadtime and the product lifetime. For this purpose we report the case with $K=50, \tau=2$ with different choices for $L$ which take the values of $0.25 \tau, 0.5 \tau$ and $0.75 \tau$. Table 3 displays our results for this setting.

For fixed $\alpha$, as the leadtime increases, $Q^{*}$ and $r^{*}$ increase, and $T^{*}$ decreases. This is to be expected because a larger leadtime increases the risk of having lost sales during this leadtime. The reordering parameters are observed to be more sensitive to the changes in leadtime. The average cost rate is also increasing in leadtime.

\subsection{Comparison with $(Q, r)$ policy}

As we propose a new policy for controlling perishable inventories, it is of interest to compare the performance of the $(Q, r, T)$ policy with that of the conventional $(Q, r)$ policy. For this purpose, we tested the two policies over a wide range of parameter settings. We report our findings on the representative cases of low and relatively high demand rates $(\lambda=0.25,5)$ for different values of $\tau, p$ and $\alpha$. The fixed parameters are $K=50, L=1$ and $h=1$. In Tables 4 and 5, we present the optimal parameters, the corresponding expected cost rates of the two policies, and the percentage improvement in the average cost rate under the $(Q, r, T)$ policy over the optimal cost under the $(Q, r)$ policy. Note that the improvement obtained by the $(Q, r, T)$ policy is higher when $\alpha$ is low $(\alpha<0.02)$ which corresponds to high service levels. When the service level constraint is tight, the $(Q, r)$ policy increases $r$ in order not to take the risk of losing sales during the leadtime. However, the $(Q, r, T)$ policy operates with smaller $r$ and chooses to order proactively at time $T$ which results in lower average costs. As the service level constraint gets looser, superiority of the $(Q, r, T)$ policy diminishes. 
Table 4

$(Q, r, T)$ vs. $(Q, r)(\lambda=0.25)$

\begin{tabular}{|c|c|c|c|c|c|c|c|c|c|c|}
\hline & & $\alpha$ & $Q_{1}^{*}$ & $r_{1}^{*}$ & $T^{*}$ & $C_{1}$ & $Q_{2}^{*}$ & $r_{2}^{*}$ & $C_{2}$ & $\Delta \%$ \\
\hline \multirow[t]{15}{*}{$p=1$} & \multirow[t]{5}{*}{$\tau=12$} & 0.005 & 4 & 1 & 9.84 & 8.19 & 5 & 4 & 11.11 & 26.28 \\
\hline & & 0.01 & 4 & 1 & 11.09 & 7.93 & 3 & 2 & 9.01 & 11.99 \\
\hline & & 0.02 & 4 & 1 & 11.33 & 7.85 & 3 & 2 & 9.01 & 12.87 \\
\hline & & 0.05 & 4 & 0 & 11.34 & 7.28 & 4 & 1 & 7.64 & 4.71 \\
\hline & & 0.1 & 3 & 0 & 12.00 & 6.87 & 3 & 0 & 6.87 & 0.0 \\
\hline & \multirow[t]{5}{*}{$\tau=15$} & 0.005 & 5 & 1 & 14.02 & 7.53 & 3 & 2 & 8.53 & 11.72 \\
\hline & & 0.01 & 4 & 1 & 14.14 & 7.244 & 3 & 2 & 8.53 & 15.12 \\
\hline & & 0.02 & 4 & 1 & 14.56 & 7.17 & 4 & 2 & 8.08 & 11.26 \\
\hline & & 0.05 & 4 & 0 & 14.30 & 6.51 & 4 & 1 & 7.10 & 8.31 \\
\hline & & 0.1 & 4 & 0 & 15.00 & 6.34 & 4 & 0 & 6.34 & 0.00 \\
\hline & \multirow[t]{5}{*}{$\tau=20$} & 0.005 & 5 & 1 & 19.00 & 6.80 & 4 & 2 & 7.67 & 11.34 \\
\hline & & 0.01 & 4 & 1 & 19.31 & 6.70 & 5 & 2 & 7.59 & 11.73 \\
\hline & & 0.02 & 4 & 1 & 20.00 & 6.66 & 4 & 1 & 6.66 & 0.00 \\
\hline & & 0.05 & 4 & 0 & 19.11 & 7.85 & 4 & 1 & 6.66 & 12.16 \\
\hline & & 0.1 & 4 & 0 & 20.00 & 5.76 & 4 & 0 & 5.76 & 0.00 \\
\hline \multirow[t]{15}{*}{$p=10$} & \multirow[t]{5}{*}{$\tau=12$} & 0.005 & 4 & 1 & 9.84 & 9.29 & 5 & 4 & 12.78 & 27.31 \\
\hline & & 0.01 & 3 & 1 & 11.02 & 8.68 & 3 & 2 & 9.66 & 10.14 \\
\hline & & 0.02 & 3 & 1 & 11.43 & 8.60 & 3 & 2 & 9.66 & 10.97 \\
\hline & & 0.05 & 3 & 0 & 10.03 & 7.97 & 3 & 1 & 8.49 & 6.12 \\
\hline & & 0.1 & 3 & 0 & 12.00 & 7.45 & 3 & 0 & 7.45 & 0.00 \\
\hline & \multirow[t]{5}{*}{$\tau=15$} & 0.005 & 4 & 1 & 11.04 & 8.29 & 3 & 2 & 8.88 & 6.64 \\
\hline & & 0.01 & 3 & 1 & 14.02 & 7.86 & 3 & 2 & 8.88 & 11.49 \\
\hline & & 0.02 & 3 & 1 & 14.88 & 7.78 & 4 & 2 & 8.72 & 10.78 \\
\hline & & 0.05 & 4 & 0 & 14.30 & 7.13 & 4 & 1 & 7.73 & 7.76 \\
\hline & & 0.1 & 3 & 0 & 15.00 & 6.68 & 3 & 0 & 6.68 & 0.00 \\
\hline & \multirow[t]{5}{*}{$\tau=20$} & 0.005 & 5 & 1 & 19.00 & 7.28 & 4 & 2 & 7.95 & 8.43 \\
\hline & & 0.01 & 4 & 1 & 19.31 & 6.98 & 4 & 2 & 7.95 & 12.20 \\
\hline & & 0.02 & 4 & 1 & 20.00 & 6.93 & 4 & 1 & 6.93 & 0.00 \\
\hline & & 0.05 & 4 & 0 & 19.11 & 6.11 & 4 & 1 & 6.93 & 11.83 \\
\hline & & 0.1 & 4 & 0 & 20.00 & 6.01 & 4 & 0 & 6.01 & 0.00 \\
\hline \multirow[t]{15}{*}{$p=50$} & \multirow[t]{5}{*}{$\tau=12$} & 0.005 & 2 & 1 & 1.12 & 11.84 & 5 & 4 & 20.02 & 41.39 \\
\hline & & 0.01 & 2 & 1 & 3.47 & 11.42 & 3 & 2 & 12.53 & 8.86 \\
\hline & & 0.02 & 2 & 1 & 11.58 & 10.91 & 3 & 2 & 12.53 & 12.93 \\
\hline & & 0.05 & 2 & 0 & 4.65 & 10.57 & 2 & 1 & 10.88 & 2.85 \\
\hline & & 0.1 & 2 & 0 & 10.92 & 9.42 & 3 & 0 & 10.06 & 6.36 \\
\hline & \multirow[t]{5}{*}{$\tau=15$} & 0.005 & 3 & 1 & 5.77 & 10.22 & 3 & 2 & 10.47 & 2.39 \\
\hline & & 0.01 & 3 & 1 & 14.02 & 9.44 & 3 & 2 & 10.47 & 9.84 \\
\hline & & 0.02 & 3 & 1 & 14.88 & 9.34 & 2 & 1 & 9.68 & 3.51 \\
\hline & & 0.05 & 3 & 0 & 10.94 & 8.77 & 3 & 1 & 9.33 & 6.00 \\
\hline & & 0.1 & 3 & 0 & 15.00 & 8.13 & 3 & 0 & 8.13 & 0.00 \\
\hline & \multirow[t]{5}{*}{$\tau=20$} & 0.005 & 3 & 1 & 6.29 & 8.59 & 3 & 2 & 8.89 & 3.37 \\
\hline & & 0.01 & 3 & 1 & 19.08 & 7.85 & 3 & 2 & 8.89 & 11.70 \\
\hline & & 0.02 & 3 & 1 & 20.00 & 7.82 & 3 & 1 & 7.82 & 0.00 \\
\hline & & 0.05 & 4 & 0 & 19.11 & 7.28 & 3 & 1 & 7.82 & 6.91 \\
\hline & & 0.1 & 3 & 0 & 20.00 & 6.63 & 3 & 0 & 6.63 & 0.00 \\
\hline
\end{tabular}

When $\alpha$ is low, parameters of the $(Q, r)$ policy are not sensitive to changes in $p$. On the other hand, those of the $(Q, r, T)$ policy depend highly on the value of $p$. The flexible nature of the $(Q, r, T)$ policy exploits better the trade-off between the tight service level constraint and the risk of incurring high perishing costs. 
Table 5

$(Q, r, T)$ vs. $(Q, r)(\lambda=5)$

\begin{tabular}{|c|c|c|c|c|c|c|c|c|c|c|}
\hline & & $\alpha$ & $Q_{1}^{*}$ & $r_{1}^{*}$ & $T^{*}$ & $C_{1}$ & $Q_{2}^{*}$ & $r_{2}^{*}$ & $C_{2}$ & $\Delta \%$ \\
\hline \multirow[t]{15}{*}{$p=1$} & \multirow[t]{5}{*}{$\tau=2$} & 0.005 & 13 & 9 & 1.00 & 37.24 & 11 & 10 & 38.67 & 3.70 \\
\hline & & 0.01 & 12 & 8 & 0.94 & 36.22 & 12 & 10 & 38.15 & 5.06 \\
\hline & & 0.02 & 12 & 7 & 1.00 & 35.22 & 10 & 8 & 36.74 & 4.14 \\
\hline & & 0.05 & 11 & 5 & 0.98 & 33.45 & 10 & 7 & 34.95 & 4.29 \\
\hline & & 0.1 & 11 & 2 & 1.05 & 31.48 & 12 & 7 & 32.41 & 2.87 \\
\hline & \multirow[t]{5}{*}{$\tau=4$} & 0.005 & 21 & 8 & 2.95 & 27.56 & 17 & 9 & 28.16 & 2.13 \\
\hline & & 0.01 & 20 & 7 & 2.81 & 26.70 & 16 & 8 & 27.38 & 2.48 \\
\hline & & 0.02 & 20 & 6 & 3.01 & 25.61 & 16 & 7 & 26.26 & 2.48 \\
\hline & & 0.05 & 19 & 4 & 2.99 & 23.96 & 18 & 6 & 24.58 & 2.52 \\
\hline & & 0.1 & 18 & 2 & 3.02 & 22.32 & 17 & 4 & 22.50 & 0.80 \\
\hline & \multirow[t]{5}{*}{$\tau=6$} & 0.005 & 24 & 8 & 4.79 & 26.04 & 22 & 9 & 26.90 & 3.20 \\
\hline & & 0.01 & 24 & 7 & 4.57 & 25.17 & 22 & 8 & 25.88 & 2.74 \\
\hline & & 0.02 & 23 & 6 & 4.61 & 24.08 & 22 & 7 & 24.86 & 3.14 \\
\hline & & 0.05 & 324 & 4 & 4.76 & 22.57 & 21 & 5 & 22.85 & 1.23 \\
\hline & & 0.1 & 20 & 3 & 6.00 & 20.92 & 20 & 3 & 20.92 & 0.00 \\
\hline \multirow[t]{15}{*}{$p=10$} & \multirow[t]{5}{*}{$\tau=2$} & 0.005 & 10 & 9 & 0.23 & 45.01 & 11 & 10 & 47.65 & 5.54 \\
\hline & & 0.01 & 9 & 8 & 0.11 & 43.28 & 10 & 9 & 44.58 & 2.92 \\
\hline & & 0.02 & 9 & 7 & 0.30 & 42.09 & 9 & 8 & 42.47 & 0.89 \\
\hline & & 0.05 & 9 & 6 & 0.84 & 39.53 & 9 & 7 & 40.75 & 2.99 \\
\hline & & 0.1 & 9 & 5 & 1.16 & 36.90 & 9 & 6 & 38.55 & 4.28 \\
\hline & \multirow[t]{5}{*}{$\tau=4$} & 0.005 & 16 & 9 & 4.00 & 29.63 & 16 & 9 & 29.63 & 0.00 \\
\hline & & 0.01 & 16 & 8 & 4.00 & 28.54 & 16 & 8 & 28.54 & 0.00 \\
\hline & & 0.02 & 16 & 7 & 4.00 & 27.41 & 16 & 7 & 27.41 & 0.00 \\
\hline & & 0.05 & 16 & 5 & 3.02 & 25.34 & 16 & 6 & 26.22 & 3.36 \\
\hline & & 0.1 & 16 & 3 & 2.89 & 23.58 & 15 & 4 & 23.72 & 0.59 \\
\hline & \multirow[t]{5}{*}{$\tau=6$} & 0.005 & 22 & 8 & 3.65 & 26.48 & 21 & 9 & 27.11 & 2.32 \\
\hline & & 0.01 & 22 & 7 & 3.70 & 25.61 & 21 & 8 & 26.09 & 1.84 \\
\hline & & 0.02 & 22 & 6 & 4.10 & 24.47 & 21 & 7 & 25.06 & 2.35 \\
\hline & & 0.05 & 22 & 4 & 4.05 & 22.97 & 20 & 5 & 22.99 & 0.09 \\
\hline & & 0.1 & 20 & 3 & 6.00 & 21.02 & 20 & 3 & 21.02 & 0.00 \\
\hline \multirow[t]{15}{*}{$p=50$} & \multirow[t]{5}{*}{$\tau=2$} & 0.005 & 10 & 9 & 0.23 & 73.46 & 11 & 10 & 87.54 & 16.08 \\
\hline & & 0.01 & 9 & 8 & 0.11 & 62.42 & 10 & 9 & 72.99 & 14.48 \\
\hline & & 0.02 & 8 & 7 & 0.05 & 54.60 & 9 & 8 & 61.50 & 11.22 \\
\hline & & 0.05 & 7 & 6 & 0.10 & 49.18 & 8 & 7 & 53.15 & 7.47 \\
\hline & & 0.1 & 7 & 5 & 0.50 & 45.79 & 7 & 6 & 47.73 & 4.06 \\
\hline & \multirow[t]{5}{*}{$\tau=4$} & 0.005 & 13 & 9 & 4.00 & 31.78 & 13 & 9 & 31.78 & 0.00 \\
\hline & & 0.01 & 13 & 8 & 4.00 & 30.69 & 13 & 8 & 30.69 & 0.00 \\
\hline & & 0.02 & 13 & 7 & 4.00 & 29.52 & 13 & 7 & 29.52 & 0.00 \\
\hline & & 0.05 & 13 & 5 & 1.79 & 27.81 & 13 & 6 & 28.25 & 1.56 \\
\hline & & 0.1 & 13 & 4 & 3.19 & 25.48 & 14 & 4 & 25.80 & 1.24 \\
\hline & \multirow[t]{5}{*}{$\tau=6$} & 0.005 & 20 & 8 & 2.88 & 27.16 & 19 & 9 & 27.51 & 1.27 \\
\hline & & 0.01 & 20 & 7 & 2.99 & 26.29 & 19 & 8 & 26.48 & 0.72 \\
\hline & & 0.02 & 20 & 6 & 3.30 & 25.15 & 19 & 7 & 25.44 & 1.14 \\
\hline & & 0.05 & 19 & 5 & 6.00 & 23.32 & 19 & 5 & 23.32 & 0.00 \\
\hline & & 0.1 & 19 & 3 & 4.61 & 21.37 & 20 & 3 & 21.46 & 0.42 \\
\hline
\end{tabular}

We observe the highest percentage difference in the average costs of the two policies when the lifetime of items is short and the service level is high. For these cases, parameters of the $(Q, r)$ policy do not change as $p$ increases. The reason for this phenomenon is the one order outstanding assumption. The $(Q, r)$ policy keeps $r$ as high as possible in order to satisfy the constraint on service level and hence operates with large $Q$. But 
the $(Q, r, T)$ policy can easily satisfy this assumption by keeping $r$ low and placing orders proactively. Thus it can decrease the value of $Q$ in order to avoid the risk of perishing especially when $p$ is large. For large $p$, an improvement of more than $40 \%$ may be obtained.

We do not observe a monotonic behavior in the percentage differences between the policies with respect to $\tau, p$ and $\alpha$. The reason for this is that the $(Q, r)$ policy selects the optimal values of $Q$ and $r$ from a discrete space. For instance, when $\tau=20$ and $\alpha=0.02$, the average costs for the two policies are the same. When we increase $\alpha$ to $5 \%$, the percentage deviation between the two policies becomes $12.16 \%$. Here we observe that the $(Q, r)$ policy is the same for both $\alpha$ values, that is it cannot find any better values for $Q$ and $r$. However, as $T$ is a continuous variable $(Q, r, T)$ policy attains a lower average cost by adjusting $T$. The main conclusions of our experimental study can be summarized as follows. The $(Q, r, T)$ policy outperforms the classical $(Q, r)$ policy in 140 experimental points out of 180 . The maximum savings are observed for the cases where the demand for items is low and the service level is high. The mean improvement for these cases is approximately $12 \%$. Some cases of high desired service levels in the experimental setup are not even feasible under the $(Q, r)$ policy. The introduction of an age threshold for reorder becomes less effective in reducing costs as the shelflife of products gets longer as expected.

\section{Conclusions and future work}

In this paper, we have proposed a control policy for perishables which bases reordering decisions not only on the current level of inventory but also explicitly on the remaining shelflife of the items in stock. Under this policy, we have developed a lost sales perishable inventory model that is suitable for environments where the items in a batch are preserved without deterioration until the batch is unpacked for usage.

The proposed $(Q, r, T)$ policy has been observed to be superior to the conventional $(Q, r)$ policy which ignores the lifetime status of items in stock. The proposed policy performs particularly well under tight service level constraints for slow moving items with short lifetimes.

A generalization of our work would be to allow the aging of the batches to start as soon as they join the stock. In this case, since the items start to age immediately after the order is received, at the instance when the inventory level hits $Q$, the items in the batch may not be fresh with the remaining shelflives being random due to demand realizations after the order placement. Hence, such instances do not constitute regeneration points as in our model and a single age threshold policy may not be appropriate. An age-based policy in the spirit of the one proposed herein would consist of a surface of age thresholds that depends on the remaining lifetimes of the items as a new batch is unpacked. Similarly, the introduction of backordering into our model would call for a more complex policy structure.

\section{Acknowledgement}

This research is partially supported by TUBITAK MISAG-104 Grant.

\section{Appendix A}

\section{A.1. Derivation of (2)}

Letting $I(\cdot)$ denote the indicator function of the event stated in the argument, the expected cycle length is given by 


$$
\begin{aligned}
E[C L]= & E\left[( X _ { Q - r } + L ) I \left(X_{Q-r}<T, X_{Q}<\min \left(X_{Q-r},+L, \tau\right)\right.\right. \\
& \text { or } \left.\left.X_{Q-r}<T, \tau<\min \left(X_{Q}, X_{Q-r}+L\right)\right)\right] \\
& +E\left[\left(X_{Q-r}+L\right) I\left(\tau-L<X_{Q-r}<T, X_{Q}>\tau\right)\right] \\
& +E\left[X_{Q} I\left(X_{Q-r}<T, X_{Q-r}+L<X_{Q}<\tau\right)\right] \\
& +E\left[\tau I\left(X_{Q-r}<\tau-L, X_{Q}>\tau\right)\right]+E\left[(T+L) I\left(T<X_{Q-r}<\tau\right)\right] .
\end{aligned}
$$

Then, noting that we can express $X_{Q}$ as the sum of two independent components as $X_{Q}=X_{Q-r}+X_{r}$, we have

$$
\begin{aligned}
E[C L]= & \int_{x=0}^{\tau-L} \int_{y=0}^{L}(x+L) f_{Q-r}(x) f_{r}(y) \mathrm{d} y \mathrm{~d} x \\
& +\int_{x=\tau-L}^{T} \int_{y=0}^{\infty}(x+L) f_{Q-r}(x) f_{r}(y) \mathrm{d} y \mathrm{~d} x \\
& +\int_{x=0}^{\tau-L} \int_{y=L}^{\tau-x}(x+y) f_{Q-r}(x) f_{r}(y) \mathrm{d} y \mathrm{~d} x \\
& +\tau \int_{x=0}^{\tau-L} \int_{y=\tau-x}^{\infty} f_{Q-r}(x) f_{r}(y) \mathrm{d} y \mathrm{~d} x+(T+L) \int_{x=T}^{\tau} f_{Q-r}(x) \mathrm{d} x,
\end{aligned}
$$

which can be written as

$$
\begin{aligned}
E[C L]= & \frac{Q-r}{\lambda} F_{r}(L) F_{Q-r+1}(\tau-L)+L F_{r}(L)-F_{Q-r}(\tau-L) \\
& +\frac{Q-r}{\lambda} \int_{\tau-L}^{T} F_{r}(\tau-x) f_{Q-r+1}(x) \mathrm{d} x+L \int_{\tau-L}^{T} F_{r}(\tau-x) f_{Q-r}(x) \mathrm{d} x \\
& +\frac{Q-r}{\lambda}\left[F_{Q-r+1}(T)-F_{Q-r+1}(\tau-L)\right]-\frac{Q-r}{\lambda} \int_{\tau-L}^{T} F_{r}(\tau-x) f_{Q-r+1}(x) \mathrm{d} x \\
& +L\left[F_{Q-r}(T)-F_{Q-r}(\tau-L)\right] \\
& -L \int_{\tau-L}^{T} F_{r}(\tau-x) f_{Q-r}(x) \mathrm{d} x+\frac{Q-r}{\lambda} \int_{0}^{\tau-L} F_{r}(\tau-x) f_{Q-r+1}(x) \mathrm{d} x \\
& -\frac{Q-r}{\lambda} F_{r}(L) F_{Q-r+1}(\tau-L)+\frac{r}{\lambda} \int_{0}^{\tau-L} F_{r+1}(\tau-x) f_{Q-r}(x) \mathrm{d} x \\
& -\frac{r}{\lambda} F_{r+1}(L) F_{Q-r}(\tau-L)+\tau F_{Q-r}(\tau-L) \\
& -\tau \int_{0}^{\tau-L} F_{r}(\tau-x) f_{Q-r}(x) \mathrm{d} x+(T+L)\left[1-F_{Q-r}(T)\right] .
\end{aligned}
$$


Finally, we can simplify it as

$$
\begin{aligned}
E[C L]= & L+T \bar{F}_{Q-r}(T)+\frac{Q-r}{\lambda} \int_{0}^{\tau-L} F_{r}(\tau-x) f_{Q-r+1}(x) \mathrm{d} x \\
& +\frac{r}{\lambda} \int_{0}^{\tau-L} F_{r+1}(\tau-x) f_{Q-r}(x) \mathrm{d} x-\tau \int_{0}^{\tau-L} F_{r}(\tau-x) f_{Q-r}(x) \mathrm{d} x \\
& +L\left[F_{r}(L)-\frac{r}{\lambda} F_{r+1}(L)+\tau-L\right] F_{Q-r}(\tau-L) \\
& +\frac{Q-r}{\lambda}\left[F_{Q-r+1}(T)-F_{Q-r+1}(\tau-L)\right] .
\end{aligned}
$$

The result is obtained after some algebraic manipulation.

\section{A.2. Derivation of (4)}

Expected value of (3) is written as

$$
\begin{aligned}
E[O H]= & E\left[\sum_{i=1}^{N(\tau)} X_{i} I\left(X_{Q}>\tau\right)\right]+E\left[\sum_{i=1}^{Q} X_{i} I\left(X_{Q}<\tau\right)\right] \\
& +E\left[\sum_{i=1}^{N(\tau)} X_{i} I\left(X_{Q}>\tau\right)\right]+E\left[\tau(Q-N(\tau)) I\left(X_{Q}>\tau\right)\right] \\
& +E\left[Q\left(X_{Q}-X_{Q-r}-L\right) I\left(X_{Q-r}<T, X_{Q-r}+L<X_{Q}<\tau\right)\right] \\
& +E\left[Q\left(\tau-X_{Q-r}-L\right) I\left(X_{Q-r}<\tau-L, X_{Q}>\tau\right)\right] .
\end{aligned}
$$

Expanding each of the terms separately, we get

$$
\begin{aligned}
E[O H]_{1} & =E\left[\sum_{i=1}^{N(\tau)} X_{i} I\left(X_{Q}>\tau\right)\right]=E\left[\sum_{i=1}^{N(\tau)} X_{i} I(N(\tau)<Q)\right] \\
& =E\left[E \sum_{i=1}^{N(\tau)} X_{i} I(N(\tau)<Q) \mid N(\tau)=n\right] \\
& =\sum_{i=1}^{Q-1} n \frac{\tau}{2} \mathrm{e}^{-\lambda \tau} \frac{(\lambda \tau)^{n}}{n !}=\frac{\lambda \tau^{2}}{2}\left[1-F_{Q-1}(\tau)\right] .
\end{aligned}
$$

Noting that the joint density of $\left(X_{1}, X_{2}, \ldots, x_{Q}\right)$ is given by

$$
f\left(x_{1}, x_{2}, \ldots, x_{Q}\right)=\lambda \mathrm{e}^{-\lambda x_{1}} \lambda \mathrm{e}^{-\lambda\left(x_{2}-x_{1}\right)} \ldots \lambda \mathrm{e}^{-\lambda\left(x_{Q}-x_{Q-1}\right)}=\lambda^{Q} \mathrm{e}^{-\lambda x_{Q}},
$$


we have

$$
\begin{aligned}
E[O H]_{2}= & E\left[\sum_{i=1}^{Q} X_{i} I\left(X_{Q}<\tau\right)\right] \\
= & \int_{x_{Q}=0}^{\tau} \int_{x_{Q-1}=0}^{x_{Q}} \cdots \int_{x_{1}=0}^{x_{2}}\left(x_{1}+x_{2}+\cdots+x_{Q}\right) \lambda^{Q} \mathrm{e}^{-\lambda x_{Q}} \mathrm{~d} x_{1} \mathrm{~d} x_{2} \cdots \mathrm{d} x_{Q} \\
= & \sum_{i=1}^{Q} \int_{x_{Q}=0}^{\tau} \int_{x_{Q-1}=0}^{x_{Q}} \cdots \int_{x_{i}=0}^{x_{i+1}} \cdots \int_{x_{1}=0}^{x_{2}} x_{i} \lambda^{Q} \mathrm{e}^{-\lambda x_{Q}} \mathrm{~d} x_{1} \cdots \mathrm{d} x_{i} \cdots \mathrm{d} x_{Q} \\
= & \sum_{i=1}^{Q} \frac{i}{\lambda} F_{Q+1}(\tau)=\frac{Q(Q+1)}{2 \lambda} F_{Q+1}(\tau), \\
E[O H]_{3}= & E\left[\tau(Q-N(\tau)) I\left(X_{Q}>\tau\right)\right] \\
= & Q \tau\left[1-F_{Q}(\tau)\right]-\tau E[N(\tau) I(N(\tau)<Q)] \\
= & Q \tau\left[1-F_{Q}(\tau)\right]-\lambda \tau^{2}\left[1-F_{a Q-1}(\tau)\right], \\
E[O H]_{4}= & E\left[Q\left(X_{Q}-X_{Q-r}-L\right) I\left(X_{Q-r}<T, X_{Q-r}+L<X_{Q}<\tau\right)\right] \\
= & Q \int_{x=0}^{\tau-L} \int_{y=L}^{\tau-x}(y-L) f_{Q-r}(x) f_{r}(y) \mathrm{d} y \mathrm{~d} x \\
= & Q \frac{r}{\lambda} \int_{x=0}^{\tau-L} F_{r+1}(\tau-x) f_{Q-r}(x) \mathrm{d} x \\
= & Q \frac{r}{\lambda} F_{r+1}(L) F_{Q-r}(\tau-L)+Q L F_{r}(L) F_{Q-r}(\tau-L) \\
& -Q L \int_{x=0}^{\tau-L} F_{r}(\tau-x) f_{Q-r}(x) \mathrm{d} x . \\
& \\
& \\
&
\end{aligned}
$$

Finally

$$
\begin{aligned}
E[O H]_{5}= & E\left[Q\left(\tau-X_{Q-r}-L\right) I\left(X_{Q-r}<\tau-L, X_{Q}>\tau\right)\right] \\
= & Q(\tau-L) \int_{x=0}^{\tau-L} \int_{y=\tau-x}^{\infty} f_{Q-r}(x) f_{r}(y) \mathrm{d} y \mathrm{~d} x \\
& -Q \int_{x=0}^{\tau-L} \int_{y=\tau-x}^{\infty} x f_{Q-r}(x) f_{r}(y) \mathrm{d} y \mathrm{~d} x \\
= & Q(\tau-L) F_{Q-r}(\tau-L)-Q(\tau-L) \int_{x=0}^{\tau-L} f_{Q-r}(x) F_{r}(\tau-x) \mathrm{d} x \\
& -Q \frac{Q-r}{\lambda} F_{Q-r+1}(\tau-L)+Q \frac{Q-r}{\lambda} \int_{x=0}^{\tau-L} f_{Q-r+1}(x) F_{r}(\tau-x) \mathrm{d} x .
\end{aligned}
$$

The result is obtained by summing the above terms. 


\section{A.3. Derivation of (9)}

We start with evaluating the expected cycle length. Observe that we can write

$$
C L= \begin{cases}X_{Q} & X_{Q-r}<T, X_{Q-r}+L<X_{Q}<\tau \\ & \text { or } X_{Q-r}>T, T+L<X_{Q}<\tau, \\ X_{Q-r}+L & X_{Q-r}<T, X_{Q-r}<X_{Q}>X_{Q-r}+L, \\ \tau & X_{Q}>\tau, \\ T+L & T<X_{Q-r}<X_{Q}<T+L,\end{cases}
$$

so that, after integration we have

$$
\begin{aligned}
E[C L]= & \int_{x=0}^{T} \int_{y=L}^{\tau-x}(x+y) f_{Q-r}(x) f_{r}(y) \mathrm{d} y \mathrm{~d} x \\
& +\int_{x=T}^{T+L} \int_{y=T+L-x}^{\tau-x}(x+y) f_{Q-r}(x) f_{r}(y) \mathrm{d} y \mathrm{~d} x \\
& +\int_{x=T+L}^{\tau} \int_{y=0}^{\tau-x}(x+y) f_{Q-r}(x) f_{r}(y) \mathrm{d} y \mathrm{~d} x \\
& +\int_{x=0}^{T} \int_{y=0}^{L}(x+L) f_{Q-r}(x) f_{r}(y) \mathrm{d} y \mathrm{~d} x \\
& +\tau E\left[I\left(X_{Q}>\tau\right)\right]+(T+L) \int_{x=T}^{T+L} \int_{y=0}^{T+L-x} f_{Q-r}(x) f_{r}(y) \mathrm{d} y \mathrm{~d} x
\end{aligned}
$$

and evaluation of the integrals results in

$$
\begin{aligned}
E[C L]= & \frac{Q}{\lambda} F_{Q+1}(\tau)+\tau\left[1-F_{Q}(\tau)\right]-\frac{Q-r}{\lambda} \int_{T}^{T+L} F_{r}(T+L-x) f_{Q-r+1}(x) \mathrm{d} x \\
& +(T+L) \int_{T}^{T+L} F_{r}(T+L-x) f_{Q-r}(x) \mathrm{d} x+\left[L F_{r}(L)-\frac{r}{\lambda} F_{r+1}(L)\right] F_{Q-r}(T) \\
& -\frac{r}{\lambda} \int_{T}^{T+L} F_{r+1}(T+L-x) f_{Q-r}(x) \mathrm{d} x .
\end{aligned}
$$

A.4. Derivation of (10)

Writing

$$
\mathrm{OH}= \begin{cases}\sum_{i=1}^{Q} X_{i}+Q\left[X_{Q}-X_{Q-r}-L\right] & X_{Q-r}<T, X_{Q-r}+L<X_{Q}<\tau, \\ \sum_{i=1}^{Q} X_{i} & X_{Q-r}<T, X_{Q}>X_{Q-r}+L \\ & \text { or } X_{Q-r}>T, X_{Q}<T+L, \\ \sum_{i=1}^{N(\tau)} X_{i}+\tau[Q-N(\tau)]+Q\left[\tau-X_{Q-r}-L\right] & X_{Q-r}<T, X_{Q}>\tau, \\ \sum_{i=1}^{N(\tau)} X_{i}+\tau[Q-N(T)+Q[\tau-T-L] & X_{Q-r}>T, X_{Q}>\tau, \\ \sum_{i=1}^{Q} X_{i}+Q\left[X_{Q}-T-L\right] & X_{Q-r}>T, T+L<X_{Q}<\tau,\end{cases}
$$


we have

$$
\begin{aligned}
E[O H]= & E\left[\sum_{i=1}^{N(\tau)} X_{i} I\left(X_{Q}>\tau\right)\right]+E\left[\sum_{i=1}^{Q} X_{i} I\left(X_{Q}<\tau\right)\right] \\
& +E\left[Q\left(X_{Q}-X_{Q-r}-L\right) I\left(X_{Q-r}<T, X_{Q-r}+L<X_{Q}<\tau\right)\right] \\
& +E\left[\tau(Q-N(\tau)) I\left(X_{Q}>\tau\right)\right] \\
& +E\left[Q\left(\tau-X_{Q-r}-L\right) I\left(X_{Q-r}<T, X_{Q}>\tau\right)\right] \\
& +E\left[Q(\tau-T-L) I\left(X_{Q-r}>T, X_{Q}>\tau\right)\right] \\
& +E\left[Q\left(X_{Q}-T-L\right) I\left(X_{Q-r}>T, T+L<X_{Q}<\tau\right)\right] .
\end{aligned}
$$

We evaluate the components of the above expression separately as follows:

$$
\begin{aligned}
E[O H]_{1}= & E\left[\sum_{i=1}^{N(\tau)} X_{i} I\left(X_{Q}>\tau\right)\right]=E\left[E\left[\sum_{i=1}^{N(\tau)} X_{i} I(N(\tau)<Q \mid N(\tau)=n)\right]\right] \\
= & \sum_{n=0}^{Q-1} n \frac{\tau}{2} P(N(\tau)=n)=\frac{\tau}{2} \sum_{n=0}^{Q-1} n P(N(\tau)=n) \\
= & \frac{\tau}{2}\left[\lambda \tau-\lambda \tau \sum_{n=Q-1}^{\infty} P(N(\tau)=n)\right]=\frac{\lambda \tau^{2}}{2}\left[1-F_{Q-1}(\tau)\right] \\
E[O H]_{2}= & E\left[\sum_{i=1}^{Q} X_{i} I\left(X_{Q}<\tau\right)\right]=\frac{Q(Q+1)}{2 \lambda} F_{Q+1}(\tau), \\
E[O H]_{3}= & E\left[Q\left(X_{Q}-X_{Q-r}-L\right) I\left(X_{Q-r}<T, X_{Q-r}+L<X_{Q}<\tau\right)\right] \\
= & Q \int_{x=0}^{T} \int_{y=L}^{\tau-x}(y-L) f_{Q-r}(x) f_{r}(y) \mathrm{d} y \mathrm{~d} x \\
= & Q \int_{x=0}^{T} \int_{y=L}^{\tau-x} y f_{Q-r}(x) f_{r}(y) \mathrm{d} y \mathrm{~d} x \\
& -Q L \int_{x=0}^{T} \int_{y=L}^{\tau-x} f_{Q-r}(x) f_{r}(y) \mathrm{d} y \mathrm{~d} x \\
= & Q \tau \bar{F}_{Q}(\tau)-\tau\left[\lambda \tau-\sum_{n=Q}^{\infty} n P(N(\tau)=n)\right] \\
= & Q \tau \bar{F}_{Q}(\tau)-\lambda \tau^{2} \bar{F}_{Q-1}(\tau), \\
E[O H]_{4}= & E\left[\tau(Q-N(\tau)) I\left(X_{Q}>\tau\right)\right] \\
= & Q \tau \bar{F}_{Q}(\tau)-\tau \sum_{n=0}^{Q-1} n P(N(\tau)=n) \\
& \\
&
\end{aligned}
$$




$$
\begin{aligned}
E[O H]_{5}= & E\left[Q\left(\tau-X_{Q-r}-L\right) I\left(X_{Q-r}<T, X_{Q}>\tau\right)\right] \\
= & Q(\tau-L) \int_{x=0}^{T} \int_{y=\tau-x}^{\infty} f_{Q-r}(x) f_{r}(y) \mathrm{d} y \mathrm{~d} x \\
& -Q \int_{x=0}^{T} \int_{y=\tau-x}^{\infty} x f_{Q-r}(x) f_{r}(y) \mathrm{d} y \mathrm{~d} x \\
= & Q(\tau-L) F_{Q-r}(T)-Q(\tau-L) \int_{x=0}^{T} f_{Q-r}(x) F_{r}(\tau-x) \mathrm{d} x \\
& -Q \frac{Q-r}{\lambda} F_{Q-r+1}(T)+Q \frac{Q-r}{\lambda} \int_{x=0}^{T} f_{Q-r+1}(x) F_{r}(\tau-x) \mathrm{d} x, \\
E[O H]_{6}= & E\left[Q(\tau-T-L) I\left(X_{Q-r}>T, X_{Q}>\tau\right)\right] \\
= & Q(\tau-T-L) \int_{x=T}^{\tau} \int_{y=\tau-x}^{\infty} f_{Q-r}(x) f_{r}(y) \mathrm{d} y \mathrm{~d} x \\
& +Q(\tau-T-L) \int_{x=\tau}^{\infty} \int_{y=0}^{\infty} f_{Q-r}(x) f_{r}(y) \mathrm{d} y \mathrm{~d} x \\
= & Q(\tau-T-L)-Q(\tau-T-L) F_{Q-r}(T) \\
& -Q(\tau-T-L) \int_{x=T}^{\tau} f_{Q-r}(x)\left[1-F_{r}(\tau-x)\right] \mathrm{d} x .
\end{aligned}
$$

Collecting these terms yields the result.

\section{A.5. Derivation of (11)}

$$
L S= \begin{cases}N\left(X_{Q-r}+L-X_{Q}\right) & \text { if } X_{Q-r}<T, \quad X_{Q}<X_{Q-r}+L \\ N\left(T+L-X_{Q}\right) & \text { if } T<X_{Q-r}, \quad X_{Q}<T+L\end{cases}
$$

Taking expectations, we have

$$
\begin{aligned}
E[L S]_{1}= & E\left[N\left(X_{Q-r}+L-X_{Q}\right) I\left(X_{Q-r}<T, X_{Q}<X_{Q-r}+L\right],\right. \\
E[L S]_{2}= & E\left[N\left(T+L-X_{Q}\right) I\left(T<X_{Q-r}, X_{Q}<T+L\right)\right] \\
= & \lambda(T+L) \int_{x=T}^{T+L} \int_{y=0}^{T+L-x} f_{Q-r}(x) f_{r}(y) \mathrm{d} y \mathrm{~d} x \\
& -\lambda \int_{x=T}^{T+L} \int_{y=0}^{T+L-x} x f_{Q-r}(x) f_{r}(y) \mathrm{d} y \mathrm{~d} x \\
& -\lambda \int_{x=T}^{T+L} \int_{y=0}^{T+L-x} y f_{Q-r}(x) f_{r}(y) \mathrm{d} y \mathrm{~d} x
\end{aligned}
$$




$$
\begin{aligned}
= & \lambda(T+L) \int_{x=T}^{T+L} F_{r}(T+L-x) f_{Q-r}(x) \mathrm{d} x \\
& -(Q-r) \int_{x=T}^{T+L} F_{r}(T+L-x) f_{Q-r+1}(x) \mathrm{d} x \\
& -r \int_{x=T}^{T+L} F_{r+1}(T+L-x) f_{Q-r}(x) \mathrm{d} x .
\end{aligned}
$$

Thus

$$
\begin{aligned}
E[L S]= & F_{Q-r}(T)\left[\lambda L F_{r}(L)-r F_{r+1}(L)\right]+\lambda(T+L) \int_{x=T}^{T+L} F_{r}(T+L-x) f_{Q-r}(x) \mathrm{d} x \\
& -\int_{x=T}^{T+L}\left[r F_{r+1}(T+L-x) f_{Q-r}(x)+(Q-r) F_{r}(T+L-x) f_{Q-r+1}(x)\right] \mathrm{d} x .
\end{aligned}
$$

\section{A.6. Derivation of (12)}

$$
\begin{aligned}
E[P] & =E\left[(Q-N(\tau)) I\left(X_{Q}>\tau\right)\right] \\
& =Q\left[1-F_{Q}(\tau)\right]-\sum_{n=0}^{Q-1} n P(N(\tau)=n)=Q\left[1-F_{Q}(\tau)\right]-\lambda \tau\left[1-F_{Q-1}(\tau)\right] .
\end{aligned}
$$

Further details of the derivations in this section may be found in Tekin [30].

\section{References}

[1] H.N. Chiu, An approximation to the continuous review inventory model with perishable items and leadtimes, European Journal of Operations Research 87 (1995) 93-108.

[2] M.A. Cohen, Analysis of single critical number ordering policies for perishable inventories, Operations Research 24 (1976) 726-741.

[3] B. Fries, Optimal ordering policy for a perishable commodity with fixed lifetime, Operational Research 23 (1975) $46-61$.

[4] G.J. Hadley, T.M. Whitin, Analysis of Inventory Systems, Prentice-Hall, Englewood Cliffs, NJ, 1963.

[5] S. Kalpakam, G. Arivarignan, A continuous review perishable inventory model, Statistics 19 (1988) 389-398.

[6] S. Kalpakam, K.P. Sapna, Continuous review $(s, S)$ inventory system with random lifetimes and positive leadtimes, Operations Research Letters 16 (1994) 115-119.

[7] S. Kalpakam, K.P. Sapna, A lost sales $(S-1, S)$ perishable inventory system with renewal demand, Naval Research Logistics 43 (1996) 129-142.

[8] L. Liu, $(s, S)$ continuous review inventory models for inventory with random lifetimes, Operations Research Letters 9 (1990) 161-169.

[9] L. Liu, D. Shi, An $(s, S)$ model for inventory with exponential lifetimes and renewal demands, Naval Research Logistics 46.

[10] Z. Lian, L. Liu, A discrete-time model for perishable inventory systems, Annals of Operation Research 87 (1999) $103-116$.

[11] L. Liu, Z. Lian, $(s, S)$ continuous review model for products with fixed lifetimes, Operations Research 47 (1999) $150-158$.

[12] L. Liu, T. Yang, An $(s, S)$ random lifetime inventory model with a positive leadtime, European Journal of Operations Research 113 (1999) 52-63.

[13] A.K. Moorthy, Y.C. Narasimhulu, I.R. Basha, On perishable inventory with Markov chain demand quantities, International Journal of Information and Managment Sciences 3 (1992) 29-37.

[14] S. Nahmias, W.P. Pierskalla, Optimal ordering policies for a product that perishes in two periods subject to stochastic demand, Naval Research Logistics Quarterly 20 (1973) 207-229.

[15] S. Nahmias, Optimal ordering policies for perishable inventory, Operations Research 23 (1975) 735-749. 
[16] S. Nahmias, A comparison of alternative approximations for ordering perishable inventory, INFOR 13 (1975) $175-184$.

[17] S. Nahmias, On ordering perishable inventory when both demand and lifetime are random, Management Science 24 (1977) 82-90.

[18] S. Nahmias, The fixed-charge perishable inventory problem, Operations Research 26 (1978) 464-481.

[19] S. Nahmias, Perishable inventory theory: A review, Operations Research 30 (1982) 681-707.

[20] S. Nahmias, S. Wang, A heuristic lot size reorder point model for decaying inventories, Management Science 25 (1979) 90-97.

[21] P. Nandsakumar, T.T. Morton, Near myopic heuristics for the fixed-life perishable problem, Management Science 39 (1993) $1490-1498$.

[22] D. Perry, M.J.M. Posner, An $(S-1, S)$ inventory system with fixed shelflife and constant leadtime, Operations Research S46 (1998) 565-571.

[23] R. Peterson, E.A. Silver, Decision Systems for Inventory Management and Production Planning, Wiley, New York, 1979.

[24] F. Raafat, Survey of literature on continuously deteriorating inventory models, Operational Research Society 42 (1991) $27-37$.

[25] N. Ravichandran, Stochastic analysis of a continuous review perishable inventory system with positive leadtime and poisson demands, European Journal of Operational Research 84 (1995) 444-457.

[26] S. Ross, Stochastic Processes, Wiley, New York, 1983.

[27] C.P. Schmidt, S. Nahmias, $(S-1, S)$ policies for perishable inventory, Management Science 31 (1985) 719-728.

[28] C.R. Schultz, Replenishment delays for expensive slow-moving items, Management Science 35 (1989) $1454-1462$.

[29] E. Tekin, A time-based control policy for a perishable inventory system with lost sales, M.Sc. thesis, Department of Industrial Engineering, Bilkent University, Ankara, Turkey.

[30] G.J.J. Van Zyl, Inventory control for perishable commodities, unpublished Ph.D. dissertation, University of North Carolina, 1964.

[31] H. Weiss, Optimal ordering policies for continuous review perishable inventory models, Operations Research 28 (1980) $365-374$. 